Research Article

\title{
Knowledge, Attitudes, Beliefs and Willingness to Recommend Human Papillomavirus (HPV) Vaccination among Medical Students in Mysore, India
}

\author{
Vijaya Srinivas', Anne E Fehrenbacher ${ }^{2}$, N Shashikala $^{3}$, Sushantha Perduru ${ }^{4}$, Karl Krupp $^{1,5}$, \\ Purnima Madhivanan ${ }^{1,5,67,}$, Mudassir Khan $^{4}$ \\ ${ }^{1}$ Public Health Research Institute of India, Mysore, India. \\ ${ }^{2}$ Semel Institute for Neuroscience and Human Behaviour, University of California, Los Angeles, USA. \\ ${ }^{3}$ Dept. of Community Medicine, KVG Medical College and Hospital, Sullia, Dakshina Kannada, Karnataka, India. \\ ${ }^{4}$ Dept. of Community Medicine, Mysore Medical College and Research Institute, Mysore, Karnataka, India. \\ ${ }^{5}$ Dept. of Health Promotion Sciences, Mel and Enid Zuckerman College of Public Health, University of Arizona, Tucson, USA. \\ ${ }^{6}$ Dept. of Family \& Community Medicine, College of Medicine, University of Arizona, Tucson, USA. \\ ${ }^{7}$ Division of Infectious Diseases, College of Medicine, University of Arizona, Tucson, USA.
}

DOI: https://doi.org/10.24321/2454.325X.202015

\section{I $\quad \mathbf{N} \quad \mathbf{F} \quad \mathbf{O}$}

\author{
Corresponding Author: \\ Mudassir Khan, Department of Community \\ Medicine, Mysore Medical College and Research \\ Institute, Mysuru, Karnataka, India \\ E-mail Id: \\ mudkhan@gmail.com \\ Orcid Id: \\ https://orcid.org/0000-0002-3625-6249 \\ How to cite this article: \\ Srinivas V, Fehrenbacher AE, Shashikala N, \\ Perduru S, Krupp K, Khan M et al. Knowledge, \\ Attitudes, Beliefs and Willingness to Recommend \\ Human Papillomavirus (HPV) Vaccination among \\ Medical Students in Mysore, India. Int J Preven \\ Curat Comm Med 2020; 6(4): 9-18.
}

Date of Submission: 2021-03-11

Date of Acceptance: 2021-03-31

\section{$\begin{array}{llllllll}\mathbf{A} & \mathbf{B} & \mathbf{S} & \mathbf{T} & \mathbf{R} & \mathbf{A} & \mathbf{C} & \mathbf{T}\end{array}$}

Context: Approximately 60,000 women in India die annually from cervical cancer. India also has high rates of head and neck cancers (HNCs), with most patients presenting with advanced disease. Uptake of the human papillomavirus (HPV) vaccine, which can prevent both cervical cancer and HNCs, is low in India.

Methods and Material: Study setting was Government Medical College, Mysore and it was a Cross-sectional study.

Between January to March 2018, a web-based self-administered questionnaire was completed by 498 medical students in Mysore, India. Descriptive statistics were conducted using SPSS.

Result: Only $8 \%$ of female students and no male students were vaccinated against HPV, and most did not feel susceptible to HPV. Male students were more likely than female students to believe that HPV vaccination can encourage youth to become sexually active ( $35 \%$ vs. $15 \%$; $p<0.001)$. Furthermore, knowledge regarding HPV vaccination for preventing HNCs was no higher among 4 th versus 1 st year medical students. Knowledge, attitudes, and beliefs favourable toward HPV vaccination improved with years of study.

Conclusion: Medical education that discusses the full range of uses of HPV vaccination for cancer prevention and improves comfort with topics related to adolescent sexuality may increase future provider recommendations.

Keywords: Human papillomavirus, vaccination, cancer, medical students, India 


\section{Introduction}

Cervical cancer is the most common gynaecological cancer among women worldwide. ${ }^{1}$ There were approximately 570,600 new cases in 2018, which represented $6.6 \%$ of all female cancers. Approximately $85 \%$ of deaths occur in developing countries. ${ }^{2}$ By 2030 , over $95 \%$ of deaths from cervical cancer are expected to be in low and middle income countries. ${ }^{3}$

In South Asia, India has the highest age standardized incidence of cervical cancer at 22 per 100,000 compared to 19.2 in Bangladesh, 13 in Sri Lanka. ${ }^{4}$ Despite modest declines in the incidence of cervical cancer in India, approximately 97,000 new cases and 60,000 cancer-related deaths continue to be observed annually in India. ${ }^{5}$ Approximately 1 in 50 Indian women will have cervical cancer during their lifetime compared to one in 100 women in the U.S. and Europe. ${ }^{6}$ The annual burden of new cases in India is projected to increase to 225,000 by $2025 .^{7}$

Several vaccines are licensed for use against oncogenic HPV infection, a necessary cause of cervical cancer. ${ }^{8}$ Bivalent and quadrivalent HPV vaccines were licensed in India in 2008, a nonavalent vaccine was licensed in $2018 .{ }^{9}$ The Indian Paediatric Society recommends Gardasil ${ }^{\mathrm{TM}}$, a quadrivalent vaccine marketed by Merck, Cervarix ${ }^{\mathrm{TM}}$, a bivalent vaccine manufactured by Glaxo Smithkline. ${ }^{10}$ Both vaccines can be given as 3 doses at zero, two and six months for Gardasil, and zero, one and six months for Cervarix. Since 2006, HPV vaccines have been recommended with a change to two-dose schedule for children between the ages of nine to fourteen years with the second dose recommended at six to twelve months after the first dose. ${ }^{11}$ To date, neither vaccines are included in the Universal Immunisation Program (UIP) in Karnataka, are only available as a recommended vaccine from private healthcare providers. A doctor's recommendation has been found to increase HPV vaccine uptake..$^{12}$ In some of the states, like Punjab and Delhi, HPV vaccine program for school girls was introduced as a state level initiative. ${ }^{13}$ The vaccine is also protective for some head, neck, anal and penile cancers. ${ }^{14}$

Understanding medical students' perceptions towards HPV vaccination is crucial to implement cancer prevention programs in India successfully. This study examined medical students' perceptions of HPV vaccination and their willingness to recommend the vaccine to the parents of eligible children and adolescents.

\section{Subject and Method}

\section{Sample}

A cross-sectional study was carried out by Public Health Research Institute of India (PHRII) and Mysore Medical College and Research Institute (MMCRI). A convenience sample of students from the first to fourth year of medical training were enrolled into the study. Among the 700 students registered in 2017, 608 (87\%) agreed to be enrolled. In total, 536 ( $88 \%)$ of the selected students consented to participate in the survey.

\section{Instrument and Data Collection}

A self-administered questionnaire was used to collect data regarding HPV infection, cervical cancer, Head and Neck Cancers (HNCs) and HPV vaccination for assessing knowledge, attitudes, beliefs and willingness among the students to recommend the vaccine. The questionnaire adapted from Maharajan et al., (2015) was validated for internal consistency by conducting a pilot study with 20 participants. Based on the pilot testing, minor modifications to the questionnaire were made. ${ }^{15}$ For construction and content validity, the questionnaire was reviewed by four study investigators and two medical students before data collection. The questionnaire was administered in English, the language of instruction for medical education in India. The only exclusion criterion was unwillingness to participate in the study. The web-based questionnaire included the purpose and consent for the study. The questionnaire consisted of four sections: general information demographic data, knowledge, attitudes, beliefs about HPV, HPV vaccination and cervical cancer and HNCs and willingness to recommend the HPV vaccine. During recruitment, investigators explained the objective of the study and details about the web-based link operation. The participants were then sent the link to access and complete the questionnaire. On completion, data were saved in a cloud-based Qualtrics format and later exported and analysed in SPSS. The survey was open to students from January to March 2018. Ethical approval was obtained by the Research Ethics Committee of MMCRI and PHRII, Mysore.

\section{Data Analysis}

Pearson chi-squared tests was used to examine differences in knowledge, attitudes, beliefs and willingness to recommend the HPV vaccine between female and male students and by year of medical education. A p-value of 0.05 was used to determine significant differences.

\section{Result}

Table 1, displays the sample characteristics. Approximately half of participants were male with an average age of 20 years old. The representation of students across all four years of medical school was approximately equal. Less than $1 \%$ of students $(n=3)$ were married. The majority of students reported their religion as Hindu (88\%). While 43\% of students reported their caste as General, $41 \%$ reported belonging to Other Backward Classes (OBC), and 16\% to Scheduled Castes or Scheduled Tribes (SC/ ST). Only 17 (8\%) female students and none of the male students had been vaccinated against HPV. 
Table 2, displays the distribution of responses related to knowledge, attitudes and beliefs toward HPV vaccination and cancer prevention stratified by gender. There were no significant differences by gender for most domains. The majority of students of both genders believed that cervical cancer is a severe disease (78\%) and preventable (72\%), that HPV is extremely harmful (64\%), and that the HPV vaccination is safe (62\%) and helpful for preventing cervical cancer (76\%). Nonetheless, less than one in five students of both genders (19\%) felt susceptible to HPV. Although most (74\%) believed that accepting HPV vaccination is a good idea based on their existing knowledge, $40 \%$ were not ready to accept the HPV vaccination if offered to them today and only $53 \%$ believed that men should get the HPV vaccine.

Table I.Sociodemographic Characteristics of Medical Students in Mysore, India ( $\mathbf{N}=498)$

\begin{tabular}{|c|c|c|}
\hline Variable & Number & Percentage \\
\hline Age in years (Mean, SD) & $20.27( \pm 2.05)$ & - \\
\hline \multicolumn{3}{|l|}{$\begin{array}{c}\text { Year of Medical } \\
\text { Education }\end{array}$} \\
\hline $1^{\text {st }}$ Year & 114 & $22.9 \%$ \\
\hline $2^{\text {nd }}$ Year & 151 & $30.3 \%$ \\
\hline $3^{\text {rd }}$ Year & 123 & $24.7 \%$ \\
\hline $4^{\text {th }}$ Year & 110 & $22.1 \%$ \\
\hline \multicolumn{3}{|l|}{ Gender } \\
\hline Female & 226 & $45.4 \%$ \\
\hline Male & 272 & $54.6 \%$ \\
\hline \multicolumn{3}{|l|}{ Religion } \\
\hline Hindu & 439 & $88.2 \%$ \\
\hline Muslim & 17 & $3.4 \%$ \\
\hline Christian & 39 & $7.8 \%$ \\
\hline Other & 3 & $0.6 \%$ \\
\hline \multicolumn{3}{|l|}{ Caste } \\
\hline General & 214 & $43.0 \%$ \\
\hline OBC & 205 & $41.2 \%$ \\
\hline $\mathrm{ST} / \mathrm{SC}$ & 79 & $15.9 \%$ \\
\hline \multicolumn{3}{|l|}{ Received HPV vaccine } \\
\hline Yes & 17 & $3.4 \%$ \\
\hline No & 481 & $96.6 \%$ \\
\hline
\end{tabular}

Significant differences by gender included knowledge related to the prevention of cervical cancer and HNCs, risks of HPV vaccination, perceived importance of information for deciding whether to be vaccinated, perceptions regarding the effect of the vaccine on sexual behaviours. Female students were more likely than male students to believe that HPV vaccination is helpful for preventing cervical cancer
( $80 \%$ vs. $74 \%, p=0.026$ ). However, most students did not know that HPV vaccination could prevent HNCs, and female students were more likely than male students to be unsure ( $57 \%$ vs. $42 \%, p<0.001)$. Female students were also more likely to believe that there is less risk in being vaccinated than having HPV infection (75\% vs. $67 \%, p=0.017$ ), to report not wanting to be infected with HPV ( $85 \%$ vs. $76 \%, p=0.004)$, to believe that information on HPV is helpful for deciding whether to be vaccinated ( $86 \%$ vs. $77 \%, p=0.002$ ). Male students were more likely than female students to believe that vaccinating young people against HPV will encourage them to become sexually active ( $35 \%$ vs. $15 \%, p<0.001)$ or lead to risky sexual behaviour ( $25 \%$ vs. $15 \%, p<0.001)$.

Table 3, displays knowledge, attitudes and beliefs regarding HPV vaccination and cancer prevention stratified by number of years in medical school. Accurate knowledge and favourable attitudes and beliefs were significantly higher among students with more years of medical education for almost all questions. Additionally, the proportion of students responding that they were unsure declined with years of study indicating more confidence in responses. Large significant differences between $1^{\text {st }} v s 4^{\text {th }}$ year medical students were observed for perceived susceptibility to HPV infection ( $7 \%$ vs $37 \%, p<0.001$ ), believing that cervical cancer is preventable $(57 \%$ vs $88 \%, \mathrm{p}<0.001)$, that HPV vaccination is safe $(55 \%$ vs $81 \%, p<0.001)$ and helpful for preventing cervical cancer ( $67 \%$ vs $93 \%, p<0.001)$, that men should get the HPV vaccine $(35 \%$ vs $63 \%, p<0.001)$ and readiness to accept the HPV vaccine ( $49 \%$ vs $71 \%, \mathrm{p}<0.001$ ). However, the proportion of students who believed that HPV vaccination would not prevent HNCs was lower among $1^{\text {st }}$ vs. $4^{\text {th }}$ year students ( $6 \%$ vs. $22 \%, p<0.001)$.

Table 4, displays responses related to willingness of students to recommend HPV vaccination stratified by year in medical school. The proportion of students responding favourably was significantly higher among $3^{\text {rd }}$ and $4^{\text {th }}$ year students than $1^{\text {st }}$ and $2^{\text {nd }}$ year students for most domains, except knowing how to bring up the topic of HPV vaccination with parents, concern regarding the amount of time needed to discuss HPV vaccination, preferring to wait until a child is 15-16 years old before recommending the HPV vaccine. Willingness to discuss and recommend HPV vaccination was endorsed by the majority of medical students across all domains from the $1^{\text {st }}$ year of medical school and increased to near universal approval by $4^{\text {th }}$ year for believing that providers should use every opportunity to recommend the HPV vaccine (97\%), intention to recommend the HPV vaccine in their practice (94\%), comfort discussing benefits and risks of HPV vaccination (94\%). Comparing $1^{\text {st }}$ vs. $4^{\text {th }}$ year students, the proportion of students who reported that they would recommend giving the HPV vaccine to a 9-10 year old girl was significantly higher with years of study ( $31 \%$ vs. $49 \%, p=0.002)$, but the same pattern was 
observed for preferring to wait until the child was 15-16 years old before recommending the vaccine ( $57 \%$ vs. $69 \%$, p $=0.014)$. Although comfort in discussing HPV-related issues with parents appeared to improve across multiple domains with years of study, $32 \%$ of $4^{\text {th }}$ year students still reported discomfort discussing adolescent sexuality with parents, $42 \%$ of $4^{\text {th }}$ year students did not know how to bring up the topic of HPV vaccination with parents.

Table 2.Bivariate Analysis of Beliefs and Attitudes about HPV, Cervical Cancer and HPV Vaccination by Gender Among Medical Students in Mysore, India ( $=498)$

\begin{tabular}{|c|c|c|c|c|c|}
\hline S. No. & Question & Female $(n=226)$ & Male $(n=272)$ & Total $(\mathrm{N}=498)$ & P-value \\
\hline \multirow[t]{4}{*}{1.} & \multicolumn{4}{|c|}{ Cervical cancer is a severe disease } & \multirow{4}{*}{0.767} \\
\hline & \multicolumn{4}{|c|}{\begin{tabular}{|l|l|l|} 
& $79.2 \%$ & $77.2 \%$ \\
\end{tabular}} & \\
\hline & False & $8.4 \%$ & $10.3 \%$ & $9.4 \%$ & \\
\hline & Do Not Know & $12.4 \%$ & $12.5 \%$ & $12.4 \%$ & \\
\hline \multirow[t]{4}{*}{2.} & \multicolumn{4}{|c|}{ Cervical cancer is preventable } & \multirow{4}{*}{0.168} \\
\hline & True & $73.9 \%$ & $69.5 \%$ & $71.5 \%$ & \\
\hline & False & $7.1 \%$ & $12.1 \%$ & $9.8 \%$ & \\
\hline & Do Not Know & $19.0 \%$ & $18.4 \%$ & $18.7 \% \%$ & \\
\hline \multirow[t]{4}{*}{3.} & \multicolumn{4}{|c|}{ HPV can be extremely harmful } & \multirow{4}{*}{0.889} \\
\hline & True & $62.8 \%$ & $64.3 \%$ & $63.7 \%$ & \\
\hline & False & $15.9 \%$ & $16.2 \%$ & $16.1 \%$ & \\
\hline & Do Not Know & $21.2 \%$ & $19.5 \%$ & $20.3 \%$ & \\
\hline \multirow[t]{4}{*}{4.} & \multicolumn{4}{|c|}{ I am susceptible to HPV infection } & \multirow{4}{*}{0.252} \\
\hline & True & $17.7 \%$ & $19.5 \%$ & $18.7 \%$ & \\
\hline & False & $42.0 \%$ & $47.4 \%$ & $45.0 \%$ & \\
\hline & Do Not Know & $40.3 \%$ & $33.1 \%$ & $36.3 \%$ & \\
\hline \multirow[t]{4}{*}{5.} & \multicolumn{4}{|c|}{ I am worried about the side effects of the HPV vaccination } & \multirow{4}{*}{0.917} \\
\hline & True & $27.4 \%$ & $26.1 \%$ & $26.7 \%$ & \\
\hline & False & $44.2 \%$ & $44.1 \%$ & $44.2 \%$ & \\
\hline & Do Not Know & $28.3 \%$ & $29.8 \%$ & 29.1 & \\
\hline \multirow[t]{4}{*}{6.} & \multicolumn{4}{|c|}{ HPV vaccination is helpful to prevent cervical cancer } & \multirow{4}{*}{0.026} \\
\hline & True & $79.6 \%$ & $73.5 \%$ & $76.3 \%$ & \\
\hline & False & $1.8 \%$ & $6.6 \%$ & $4.4 \%$ & \\
\hline & Do Not Know & $18.6 \%$ & $19.9 \%$ & $19.3 \%$ & \\
\hline \multirow[t]{4}{*}{7.} & \multicolumn{4}{|c|}{ HPV vaccination is safe } & \multirow{4}{*}{0.066} \\
\hline & True & $58.4 \%$ & $65.8 \%$ & $62.4 \%$ & \\
\hline & False & $5.3 \%$ & $7.4 \%$ & $6.4 \%$ & \\
\hline & Do Not Know & $36.3 \%$ & $26.8 \%$ & $31.1 \%$ & \\
\hline \multirow[t]{4}{*}{8.} & HPV vacci & event some head & nd neck cancers & & \multirow{4}{*}{0.001} \\
\hline & True & $31.9 \%$ & $39.0 \%$ & $35.7 \%$ & \\
\hline & False & $11.1 \%$ & $19.5 \%$ & $15.7 \%$ & \\
\hline & Do Not Know & $57.1 \%$ & $41.5 \%$ & $48.6 \%$ & \\
\hline \multirow[t]{4}{*}{9.} & There is less risk & ing vaccinated th & n having HPV in & ection & \multirow{4}{*}{0.017} \\
\hline & True & $75.2 \%$ & $66.9 \%$ & $70.7 \%$ & \\
\hline & False & $4.9 \%$ & $11.8 \%$ & $8.6 \%$ & \\
\hline & Do Not Know & $19.9 \%$ & $21.3 \%$ & $20.7 \%$ & \\
\hline
\end{tabular}




\begin{tabular}{|c|c|c|c|c|c|}
\hline \multirow[t]{4}{*}{10.} & \multicolumn{4}{|c|}{ Men should get the HPV vaccine to prevent their partner from getting cervical cancer } & \multirow{4}{*}{0.066} \\
\hline & True & $48.2 \%$ & $56.3 \%$ & $52.6 \%$ & \\
\hline & False & $12.8 \%$ & $14.7 \%$ & $13.9 \%$ & \\
\hline & Do Not Know & $38.9 \%$ & $29.0 \%$ & $33.5 \%$ & \\
\hline \multirow[t]{4}{*}{11.} & HPV vaccinati & increase & exual be & & \multirow{4}{*}{0.001} \\
\hline & True & $14.6 \%$ & $25.0 \%$ & $20.3 \%$ & \\
\hline & False & $34.1 \%$ & $39.0 \%$ & $36.7 \%$ & \\
\hline & Do Not Know & $51.3 \%$ & $36.0 \%$ & $43.0 \%$ & \\
\hline \multirow[t]{4}{*}{12.} & Vaccinating young peop & Il encour & to becor & Illy active & \multirow{4}{*}{0.001} \\
\hline & True & $14.6 \%$ & $34.6 \%$ & $25.5 \%$ & \\
\hline & False & $36.7 \%$ & $37.1 \%$ & $36.9 \%$ & \\
\hline & Do Not Know & $48.7 \%$ & $28.3 \%$ & $37.6 \%$ & \\
\hline \multirow[t]{4}{*}{13.} & I an & afety of $t$ & ccine & & \multirow{4}{*}{0.507} \\
\hline & True & $36.3 \%$ & $32.0 \%$ & $33.9 \%$ & \\
\hline & False & $35.8 \%$ & $40.4 \%$ & $38.4 \%$ & \\
\hline & Do Not Know & $27.9 \%$ & $27.6 \%$ & $27.7 \%$ & \\
\hline \multirow[t]{4}{*}{14.} & I believe that & Is should & he HPV & & \multirow{4}{*}{0.211} \\
\hline & True & $69.0 \%$ & $67.6 \%$ & $68.3 \%$ & \\
\hline & False & $4.9 \%$ & $8.8 \%$ & $7.0 \%$ & \\
\hline & Do Not Know & $26.1 \%$ & $23.5 \%$ & $2.4 \%$ & \\
\hline \multirow[t]{4}{*}{15.} & & be infec & APV & & \multirow{4}{*}{0.004} \\
\hline & True & $85.0 \%$ & $76.1 \%$ & $80.1 \%$ & \\
\hline & False & $1.8 \%$ & $8.1 \%$ & $5.2 \%$ & \\
\hline & Do Not Know & $13.3 \%$ & $15.8 \%$ & $14.7 \%$ & \\
\hline \multirow[t]{4}{*}{16.} & Information on HPV he & whether & e vaccin & inst HPV & \multirow{4}{*}{0.002} \\
\hline & True & $85.8 \%$ & $76.1 \%$ & $80.5 \%$ & \\
\hline & False & $1.3 \%$ & $7.4 \%$ & $4.6 \%$ & \\
\hline & Do Not Know & $12.8 \%$ & $16.5 \%$ & $14.9 \%$ & \\
\hline \multirow[t]{4}{*}{17.} & Based on my & ing HPV & $n$ is a go & & \multirow{4}{*}{0.289} \\
\hline & True & $73.5 \%$ & $74.6 \%$ & $74.1 \%$ & \\
\hline & False & $4.0 \%$ & $6.6 \%$ & $5.4 \%$ & \\
\hline & Do Not Know & $22.6 \%$ & $18.8 \%$ & $20.5 \%$ & \\
\hline \multirow[t]{4}{*}{18.} & If I were of & cination $t$ & uld acce & & \multirow{4}{*}{0.072} \\
\hline & True & $61.1 \%$ & $59.9 \%$ & $60.4 \%$ & \\
\hline & False & $6.6 \%$ & $12.5 \%$ & $9.8 \%$ & \\
\hline & Do Not Know & $32.3 \%$ & $27.6 \%$ & $29.7 \%$ & \\
\hline
\end{tabular}

Table 3.Bivariate Analysis of Beliefs and Attitudes about HPV, Cervical Cancer and HPV Vaccination by Year of Study Among Medical Students in Mysore, India ( $\mathbf{N}=498)$

\begin{tabular}{|c|c|c|c|c|c|c|c|}
\hline S. No. & Question & $1^{\text {st }}$ Year $(n=114)$ & 2 Year $(n=151)$ & $3^{\text {rd }}$ Year $(n=123)$ & $4^{\text {th }}$ Year $(n=110)$ & Total $(n=498)$ & P value \\
\hline 1. & \multicolumn{6}{|c|}{ Cervical cancer is a severe disease } & \multirow{3}{*}{0.010} \\
\hline & True & $71.1 \%$ & $75.5 \%$ & $82.9 \%$ & $83.6 \%$ & $78.1 \%$ & \\
\hline & False & $7.0 \%$ & $12.6 \%$ & $7.3 \%$ & $10.0 \%$ & $9.4 \%$ & \\
\hline
\end{tabular}




\begin{tabular}{|c|c|c|c|c|c|c|c|}
\hline & Do Not Know & $21.9 \%$ & $11.9 \%$ & $9.8 \%$ & $6.4 \%$ & $12.4 \%$ & \\
\hline 2. & \multicolumn{6}{|c|}{ Cervical cancer is preventable } & \multirow{4}{*}{0.000} \\
\hline & True & $57.0 \%$ & $62.3 \%$ & $81.3 \%$ & $88.2 \%$ & $71.5 \%$ & \\
\hline & False & $7.0 \%$ & $15.2 \%$ & $8.9 \%$ & $6.4 \%$ & $9.8 \%$ & \\
\hline & Do Not Know & $36.0 \%$ & $22.5 \%$ & $9.8 \%$ & $5.5 \%$ & $18.7 \%$ & \\
\hline 3. & & & PV can b & ely harn & & & \multirow{4}{*}{0.000} \\
\hline & True & $66.7 \%$ & $61.6 \%$ & $67.5 \%$ & $59.1 \%$ & $63.7 \%$ & \\
\hline & False & $3.5 \%$ & $15.9 \%$ & $17.1 \%$ & $28.2 \%$ & $16.1 \%$ & \\
\hline & Do Not Know & $29.8 \%$ & $22.5 \%$ & $15.4 \%$ & $12.7 \%$ & $20.3 \%$ & \\
\hline 4. & \multicolumn{6}{|c|}{ I am susceptible to HPV infection } & \multirow{4}{*}{0.000} \\
\hline & True & $7.0 \%$ & $17.2 \%$ & $14.6 \%$ & $37.3 \%$ & $18.7 \%$ & \\
\hline & False & $46.5 \%$ & $41.1 \%$ & $54.5 \%$ & $38.2 \%$ & $45.0 \%$ & \\
\hline & Do Not Know & $46.5 \%$ & $41.7 \%$ & $30.9 \%$ & $24.5 \%$ & $36.3 \%$ & \\
\hline 5. & \multicolumn{6}{|c|}{ I am worried about the side effects of the HPV vaccination } & \multirow{4}{*}{0.000} \\
\hline & True & $23.7 \%$ & $33.1 \%$ & $27.6 \%$ & $20.0 \%$ & $26.7 \%$ & \\
\hline & False & $30.7 \%$ & $33.1 \%$ & $50.4 \%$ & $66.4 \%$ & $44.2 \%$ & \\
\hline & Do Not Know & $45.6 \%$ & $33.8 \%$ & $22.0 \%$ & $13.6 \%$ & $29.1 \%$ & \\
\hline 6. & \multicolumn{6}{|c|}{ HPV vaccination is helpful to prevent cervical cancer } & \multirow{4}{*}{0.000} \\
\hline & True & $66.7 \%$ & $66.9 \%$ & $82.1 \%$ & $92.7 \%$ & $76.3 \%$ & \\
\hline & False & $1.8 \%$ & $8.6 \%$ & $4.9 \%$ & $0.9 \%$ & $4.4 \%$ & \\
\hline & Do Not Know & $31.6 \%$ & $24.5 \%$ & $13.0 \%$ & $6.4 \%$ & $19.3 \%$ & \\
\hline 7. & \multicolumn{6}{|c|}{ HPV vaccination is safe } & \multirow{4}{*}{0.000} \\
\hline & True & $55.3 \%$ & $57.6 \%$ & $58.5 \%$ & $80.9 \%$ & $62.4 \%$ & \\
\hline & False & $2.6 \%$ & $9.3 \%$ & $8.1 \%$ & $4.5 \%$ & $6.4 \%$ & \\
\hline & Do Not Know & $42.1 \%$ & $33.1 \%$ & $33.3 \%$ & $14.5 \%$ & $31.1 \%$ & \\
\hline 8. & \multicolumn{6}{|c|}{ HPV vaccination will prevent some head and neck cancers } & \multirow{4}{*}{0.004} \\
\hline & True & $32.5 \%$ & $41.7 \%$ & $31.7 \%$ & $35.5 \%$ & $35.7 \%$ & \\
\hline & False & $6.1 \%$ & $14.6 \%$ & $20.3 \%$ & $21.8 \%$ & $15.7 \%$ & \\
\hline & Do Not Know & $61.4 \%$ & $43.7 \%$ & $48.0 \%$ & $42.7 \%$ & $48.6 \%$ & \\
\hline 9. & \multicolumn{6}{|c|}{ There is less risk involved in being vaccinated than having HPV infection } & \multirow{4}{*}{0.012} \\
\hline & True & $65.8 \%$ & $68.2 \%$ & $78.0 \%$ & $70.9 \%$ & $70.7 \%$ & \\
\hline & False & $5.3 \%$ & $7.9 \%$ & $7.3 \%$ & $14.5 \%$ & $8.6 \%$ & \\
\hline & Do Not Know & $28.9 \%$ & $23.8 \%$ & $14.6 \%$ & $14.5 \%$ & $20.7 \%$ & \\
\hline 10. & \multicolumn{6}{|c|}{ Men should get the HPV vaccine to prevent their partner from getting cervical cancer } & \multirow{4}{*}{0.000} \\
\hline & True & $35.1 \%$ & $47.7 \%$ & $65.9 \%$ & $62.7 \%$ & $52.6 \%$ & \\
\hline & False & $11.4 \%$ & $14.6 \%$ & $12.2 \%$ & $17.3 \%$ & $13.9 \%$ & \\
\hline & Do Not Know & $53.5 \%$ & $37.7 \%$ & $22.0 \%$ & $20.0 \%$ & $33.5 \%$ & \\
\hline 11. & \multicolumn{6}{|c|}{ HPV vaccination can lead to an increase in risky sexual behaviour } & \multirow{4}{*}{0.000} \\
\hline & True & $15.8 \%$ & $22.5 \%$ & $22.8 \%$ & $19.1 \%$ & $20.3 \%$ & \\
\hline & False & $14.9 \%$ & $31.1 \%$ & $43.1 \%$ & $60.0 \%$ & $36.7 \%$ & \\
\hline & Do Not Know & $69.3 \%$ & $46.4 \%$ & $34.1 \%$ & $20.9 \%$ & $43.0 \%$ & \\
\hline
\end{tabular}




\begin{tabular}{|c|c|c|c|c|c|c|c|}
\hline 12. & \multicolumn{6}{|c|}{ Vaccinating young people against HPV will encourage them to become sexually active } & \multirow{4}{*}{0.000} \\
\hline & True & $14.0 \%$ & $28.5 \%$ & $31.7 \%$ & $26.4 \%$ & $25.5 \%$ & \\
\hline & False & $24.6 \%$ & $31.1 \%$ & $41.5 \%$ & $52.7 \%$ & $36.9 \%$ & \\
\hline & Do Not Know & $61.4 \%$ & $40.4 \%$ & $26.8 \%$ & $20.9 \%$ & $37.6 \%$ & \\
\hline \multirow[t]{4}{*}{13.} & \multicolumn{6}{|c|}{ I am worried about safety of the HPV vaccine } & \multirow{4}{*}{0.000} \\
\hline & True & $36.0 \%$ & $34.4 \%$ & $36.6 \%$ & $28.2 \%$ & $33.9 \%$ & \\
\hline & False & $30.7 \%$ & $29.1 \%$ & $41.5 \%$ & $55.5 \%$ & $38.4 \%$ & \\
\hline & Do Not Know & $33.3 \%$ & $36.4 \%$ & $22.0 \%$ & $16.4 \%$ & $27.7 \%$ & \\
\hline 14. & \multicolumn{6}{|c|}{ I believe that both boys and girls should receive the HPV vaccine } & \multirow{4}{*}{0.000} \\
\hline & True & $64.0 \%$ & $67.5 \%$ & $76.4 \%$ & $64.5 \%$ & $68.3 \%$ & \\
\hline & False & $0.0 \%$ & $9.3 \%$ & $4.9 \%$ & $13.6 \%$ & $7.0 \%$ & \\
\hline & Do Not Know & $36.0 \%$ & $23.2 \%$ & $18.7 \%$ & $21.8 \%$ & $24.7 \%$ & \\
\hline \multirow[t]{4}{*}{15.} & \multicolumn{6}{|c|}{ I would not want to be infected with HPV } & \multirow{4}{*}{0.077} \\
\hline & True & $78.9 \%$ & $72.8 \%$ & $84.6 \%$ & $86.4 \%$ & $80.1 \%$ & \\
\hline & False & $3.5 \%$ & $7.3 \%$ & $5.7 \%$ & $3.6 \%$ & $5.2 \%$ & \\
\hline & Do Not Know & $17.5 \%$ & $19.9 \%$ & $9.8 \%$ & $10.0 \%$ & $14.7 \%$ & \\
\hline \multirow[t]{4}{*}{16.} & Informa & on HPV & e to decic & her I sho & ccinated & HPV & \multirow{4}{*}{0.000} \\
\hline & True & $83.3 \%$ & $69.5 \%$ & $82.9 \%$ & $90.0 \%$ & $80.5 \%$ & \\
\hline & False & $0.9 \%$ & $7.9 \%$ & $6.5 \%$ & $1.8 \%$ & $4.6 \%$ & \\
\hline & Do Not Know & $15.8 \%$ & $22.5 \%$ & $10.6 \%$ & $8.2 \%$ & $14.9 \%$ & \\
\hline \multirow[t]{4}{*}{17.} & & sed on $\mathrm{m}$ & edge acc & IPV vacci & a good ic & & \multirow{4}{*}{0.000} \\
\hline & True & $67.5 \%$ & $65.6 \%$ & $80.5 \%$ & $85.5 \%$ & $74.1 \%$ & \\
\hline & False & $3.5 \%$ & $8.6 \%$ & $4.1 \%$ & $4.5 \%$ & $5.4 \%$ & \\
\hline & Do Not Know & $28.9 \%$ & $25.8 \%$ & $15.4 \%$ & $10.0 \%$ & $20.5 \%$ & \\
\hline \multirow[t]{4}{*}{18.} & & If I wa & HPV vac & n today I & ccept it & & \multirow{4}{*}{0.001} \\
\hline & True & $49.1 \%$ & $58.3 \%$ & $64.2 \%$ & $70.9 \%$ & $60.4 \%$ & \\
\hline & False & $6.1 \%$ & $11.3 \%$ & $13.8 \%$ & $7.3 \%$ & $9.8 \%$ & \\
\hline & Do Not Know & $44.7 \%$ & $30.5 \%$ & $22.0 \%$ & $21.8 \%$ & $29.7 \%$ & \\
\hline
\end{tabular}

Table 4.Bivariate Analysis of Willingness to Recommend HPV vaccination by Year of Study among Medical Students in Mysore, India ( $\mathbf{N}=498)$

\begin{tabular}{|c|c|c|c|c|c|c|c|}
\hline S. No. & Question & $1^{\text {st }}$ Year $(n=114)$ & $2^{\text {nd }} Y e a r(n=151)$ & $3^{\text {rd }}$ Year $(n=123)$ & $4^{\text {th }}$ Year $(n=110)$ & Total $(n=498)$ & $P$ value \\
\hline 1. & \multicolumn{6}{|c|}{ Providers should use every opportunity to recommend the HPV vaccine to eligible parents } & \multirow{3}{*}{0.003} \\
\hline & Agree & $81.6 \%$ & $86.8 \%$ & $94.3 \%$ & $97.3 \%$ & $89.8 \%$ & \\
\hline & Disagree & $18.4 \%$ & $13.2 \%$ & $5.7 \%$ & $2.7 \%$ & $10.2 \%$ & \\
\hline \multirow[t]{3}{*}{2.} & \multicolumn{6}{|c|}{ I am comfortable discussing adolescent sexuality with parents } & \multirow{3}{*}{0.003} \\
\hline & Agree & $51.8 \%$ & $46.4 \%$ & $65.0 \%$ & $68.2 \%$ & $57.0 \%$ & \\
\hline & Disagree & $48.2 \%$ & $53.6 \%$ & $35.0 \%$ & $31.8 \%$ & $43.0 \%$ & \\
\hline \multirow[t]{3}{*}{3.} & & I would recomm & end the HPV vacc & ine to eligible pa & ents in my practic & & \multirow{3}{*}{0.003} \\
\hline & Agree & $82.5 \%$ & $80.1 \%$ & $94.3 \%$ & $93.6 \%$ & $87.1 \%$ & \\
\hline & Disagree & $17.5 \%$ & $19.9 \%$ & $5.7 \%$ & $6.4 \%$ & $12.9 \%$ & \\
\hline \multirow[t]{2}{*}{4.} & & I don't know hol & to bring up the & opic of HPV vacc & nation with paren & & \multirow{2}{*}{0.046} \\
\hline & Agree & $34.2 \%$ & $47.7 \%$ & $41.5 \%$ & $41.8 \%$ & $41.8 \%$ & \\
\hline
\end{tabular}




\begin{tabular}{|c|c|c|c|c|c|c|c|}
\hline & Disagree & $65.8 \%$ & $52.3 \%$ & $58.5 \%$ & $58.2 \%$ & $58.2 \%$ & \\
\hline \multirow[t]{3}{*}{5.} & \multicolumn{6}{|c|}{ I am comfortable discussing the benefits and risk of HPV vaccination } & \multirow{3}{*}{0.001} \\
\hline & Agree & $79.8 \%$ & $73.5 \%$ & $85.4 \%$ & $93.6 \%$ & $82.3 \%$ & \\
\hline & Disagree & $20.2 \%$ & $26.5 \%$ & $14.6 \%$ & $6.4 \%$ & $17.7 \%$ & \\
\hline \multirow[t]{3}{*}{6.} & \multicolumn{6}{|c|}{ I prefer to wait until the child is $15-16$ years old before I recommend the HPV vaccine } & \multirow{3}{*}{0.014} \\
\hline & Agree & $59.6 \%$ & $56.3 \%$ & $70.7 \%$ & $69.1 \%$ & $63.5 \%$ & \\
\hline & Disagree & $40.4 \%$ & $43.7 \%$ & $29.3 \%$ & $30.9 \%$ & $36.5 \%$ & \\
\hline \multirow[t]{3}{*}{7.} & \multicolumn{6}{|c|}{ I am concerned about the amount of time needed to discuss HPV about vaccination with parents } & \multirow{3}{*}{0.017} \\
\hline & Agree & $43.9 \%$ & $53.6 \%$ & $55.3 \%$ & $53.6 \%$ & $51.8 \%$ & \\
\hline & Disagree & $56.1 \%$ & $46.4 \%$ & $44.7 \%$ & $46.4 \%$ & $48.2 \%$ & \\
\hline \multirow[t]{3}{*}{8.} & \multicolumn{6}{|c|}{ I would recommend the HPV vaccine to a relative } & \multirow{3}{*}{0.014} \\
\hline & Agree & $72.8 \%$ & $73.5 \%$ & $85.4 \%$ & $87.3 \%$ & $79.3 \%$ & \\
\hline & Disagree & $27.2 \%$ & $26.5 \%$ & $14.6 \%$ & $12.7 \%$ & $20.7 \%$ & \\
\hline \multirow[t]{3}{*}{9.} & \multicolumn{6}{|c|}{ I am willing to discuss HPV vaccination when parents come in for other problems } & \multirow{3}{*}{0.009} \\
\hline & Agree & $59.6 \%$ & $59.6 \%$ & $65.0 \%$ & $77.3 \%$ & $64.9 \%$ & \\
\hline & Disagree & $40.4 \%$ & $40.4 \%$ & $35.0 \%$ & $22.7 \%$ & $35.1 \%$ & \\
\hline \multirow[t]{3}{*}{10.} & I am & ng to disc & accinati & parents & or chror & tions & \multirow{3}{*}{0.000} \\
\hline & Agree & $77.2 \%$ & $64.9 \%$ & $82.9 \%$ & $79.1 \%$ & $75.3 \%$ & \\
\hline & Disagree & $22.8 \%$ & $35.1 \%$ & $17.1 \%$ & $20.9 \%$ & $24.7 \%$ & \\
\hline \multirow[t]{3}{*}{11.} & & would rec & giving $t$ & accine to & -year-ol & & \multirow{3}{*}{0.002} \\
\hline & Agree & $30.7 \%$ & $49.0 \%$ & $48.8 \%$ & $49.1 \%$ & $44.8 \%$ & \\
\hline & Disagree & $69.3 \%$ & $51.0 \%$ & $51.2 \%$ & $50.9 \%$ & $55.2 \%$ & \\
\hline
\end{tabular}

\section{Discussion}

This study examines the perspectives of Indian medical students on HPV vaccination by gender and year of medical school students' willingness to recommend HPV vaccination. A majority of participants agreed that HPV vaccination could prevent cervical cancer but were less certain about its efficacy for the prevention of HNCs, this knowledge did not appear to improve with years of study.

In this study, very few participants had received the HPV vaccine though most believed that cervical cancer was harmful. Possible reasons for this contradiction could include lack of availability of the vaccine, whether students received a provider recommendation, cost. ${ }^{16-18}$ Additionally, the disconnect between beliefs, attitudes and behaviours might be a result of uncertainty about who should receive the vaccine. ${ }^{18}$ Although approximately two-thirds of the participants agreed that HPV infection could be harmful, more than two-thirds were unsure if they were susceptible to HPV infection. These results are consistent with existing studies demonstrating medical students' limited knowledge and misperceptions about HPV infection and vaccination in India, particularly at the start of medical school. ${ }^{19-22}$

There were significant differences between female and male medical students in the study regarding the benefits of the HPV vaccine and assumptions about the effect of the vaccine on adolescent sexual behaviours. Previous studies have indicated that female students tend to be more knowledgeable about HPV and the vaccine compared to male students. ${ }^{23,24} \mathrm{~A}$ majority of participants in the current study thought the HPV vaccine was low risk, but female students were more likely to endorse this view than male students, were more likely to say that the risks of HPV infection outweighed the potential risks of the vaccine. However, male students were more likely to believe that the HPV vaccine could prevent certain HNCs, while female students were much more uncertain. The gender difference in uncertainty of the benefits of the HPV vaccine for other types of cancers could be attributed to how the HPV vaccine is marketed in India, predominantly targeting women and girls for the prevention of cervical cancer. Parents may also be more likely to accept vaccinations for girls than boys, which could influence the amount of education boys receive regarding the safety and efficacy of the vaccine for the prevention of cancers affecting both women and men. ${ }^{24}$ However, the findings might also be related to social or cultural norms regarding gender in which women are socialized to have less confidence in their knowledge than 
men, thus may question themselves more than men. ${ }^{25,26}$

Male and female students also differed in how they viewed the potential impact of the HPV vaccine on sexual behaviours, which may deter providers from recommending the vaccination to parents of young adolescents, particularly preteen girls and boys. Male students were significantly more likely to believe that HPV vaccination could increase sexual activity and risky sexual behaviours. Past studies have found similar concerns in India among medical students irrespective of gender. ${ }^{19,27}$ Sexual health is a stigmatized topic, which is rarely discussed between parents and children in India, and between providers and patients. Furthermore, while premarital sex is increasing in India, it is still not as common as in many other countries, demonstrating the stronghold of conservative attitudes towards sexual activity in India. ${ }^{28,29}$ For instance, one study highlighted how parents recognize the importance of conversations regarding sex yet do not discuss sexual health with their children. ${ }^{30}$ Social norms around sexual activity could explain why a majority of the medical students in this study, regardless of their gender or year of study, would not recommend HPV vaccination for 9-10 year old girls. In a study by Wong et al., (2010) on the role of men in promoting the uptake of HPV vaccinations, men tended to support vaccination more when it was framed paternalistically as a way to protect their spouses and daughters. ${ }^{31}$ Dispelling myths regarding spurious associations between HPV vaccination and sexual activity should be incorporated into medical education in India. A systematic review by Madhivanan et al., (2016) found no association between vaccination and sexual activity, a positive association with safer sex by precautionary measures like condom use and receipt of HPV vaccination. ${ }^{32}$ These findings are subject to limitations. First, the survey was conducted with only one medical college with a non-random sample of students, so the findings may not be generalizable to all medical students in India. Second, the responses are all self-reported, so the results are vulnerable to social desirability bias, with participants answering how they believe would be favourably viewed by investigators rather than how they truly believe or act. Nonetheless, this study with a large and diverse sample of medical students across all four years of study offers important insights regarding the role of medical education in altering knowledge and perceptions of students who will be future medical providers. This is our first web based survey to collect data regarding thec most important subject of a preventable cancer in India.

\section{Conclusion}

Further exploration of how Indian medical education curriculum addresses HPV and cancer prevention would help to bring changes in the knowledge and attitude of students which in turn help providers' recommendation for vaccine. More emphasis on the effectiveness of HPV vaccination in medical education could help to improve vaccine uptake in India.

\section{Acknowledgement}

Thanks to all the Medical students who willingly participated in this program. We also thank Shivamma N, Counsellor PHRII who worked with Qualtrix to help data analysis. Our thanks to all other staff of PHRII and Community Medicine Department of Mysore Medical College for their assistance with recruitment for this study.

\section{Conflict of Interest: None}

\section{References}

1. DeSS, Diaz M, CastellsaguéX. Worldwide prevalence and genotype distribution of cervical human papillomavirus DNA in women with normal cytology: a meta-analysis. The Lancet infectious diseases 2007; 7(7): 453-459.

2. Cervical Cancer. WHO 2019. Accessed, 2019.

3. Parkhurst JO, Vulimiri M. Cervical cancer and the global health agenda: insights from multiple policy-analysis frameworks. Global public health 2013; 8(10): 10931108.

4. Sreedevi A, Javed R, Dinesh A. Epidemiology of cervical cancer with special focus on India. International journal of women's health 2015; 7: 405.

5. Van Dyne EA. Establishing Baseline Cervical Cancer Screening Coverage, India. MMWR Morbidity and Mortality Weekly Report. 2019; 68.

6. Bobdey S, Sathwara J, Jain A, Balasubramaniam G. Burden of cervical cancer and role of screening in India. Indian journal of medical and paediatric oncology: official journal of Indian Society of Medical \& Paediatric Oncology 2016; 37(4): 278.

7. Krishnan S, Madsen E, Porterfield D, Varghese B. Advancing cervical cancer prevention in India: implementation science priorities. The oncologist 2013; 18(12): 1285.

8. Dsouza ND, Murthy N, Aras R. Projection of cancer incident cases for India-till 2026. Asian Pacific Journal of cancer prevention 2013; 14(7): 4379-4386.

9. Sankaranarayanan R, Basu P, Kaur P. Current status of human papillomavirus vaccination in India's cervical cancer prevention efforts. The Lancet Oncology 2019; 20(11): e637-e644.

10. Franco EL, Harper DM. Vaccination against human papillomavirus infection: a new paradigm in cervical cancer control. Vaccine 2005; 23(17-18): 2388-2394.

11. Kaarthigeyan K. Cervical cancer in India and HPV vaccination. Indian journal of medical and paediatric oncology: official journal of Indian Society of Medical \& Paediatric Oncology 2012; 33(1): 7.

12. Rosenthal S, Weiss TW, Zimet GD et al. Predictors 
of HPV vaccine uptake among women aged 19-26: importance of a physician's recommendation. Vaccine 2011; 29(5): 890-895.

13. Gupta M, Kaushal K, Sharma N. Newer vaccines (measles-rubella, human papillomavirus, rotavirus, and pneumococcal conjugate vaccine) introduction: Experience from Northern India. International Journal of Noncommunicable Diseases 2018; 3(5): 25.

14. Young D, Xiao CC, Murphy B et al. Increase in head and neck cancer in younger patients due to human papillomavirus (HPV). Oral oncology 2015; 51(8): 727730.

15. Maharajan MK, Rajiah K, Num K et al. Knowledge of human papillomavirus infection, cervical cancer and willingness to pay for cervical cancer vaccination among ethnically diverse medical students in Malaysia. Asian Pac J Cancer Prev 2015; 16(14): 5733-5739.

16. Small SL, Patel DA. Impact of HPV vaccine availability on uptake. The Journal for Nurse Practitioners 2012; 8(1): 61-66.

17. Basu P, Chowdhury D. Cervical cancer screening \& HPV vaccination: a comprehensive approach to cervical cancer control. Indian Journal of Medical Research 2009; 130(3): 241.

18. Padmanabha N, Kini JR, Alwani AA et al. Acceptability of human papillomavirus vaccination among medical students in Mangalore, India. Vaccine 2019; 37(9): 1174-1181.

19. Mehta S, Rajaram S, Goel G et al. Awareness about human papilloma virus and its vaccine among medical students. Indian journal of community medicine: official publication of Indian Association of Preventive \& Social Medicine 2013; 38(2): 92.

20. Swarnapriya K, Kavitha D, Reddy G. Knowledge, attitude and practices regarding HPV vaccination among medical and para medical in students, India a cross sectional study. Asian Pac J Cancer Prev 2015; 16(18): 8473-8477.

21. Canon C, Effoe V, Shetty V et al. Knowledge and attitudes towards human papillomavirus (HPV) among academic and community physicians in Mangalore, India. Journal of Cancer Education 2017; 32(2): 382-391.

22. Aamir F, Naqvi S, Farooquee A. Comparison of awareness, uptake and barriers of Human Papilloma Virus vaccine among students of private medical college in Karachi-A cross sectional study. Pak J Surg 2019; 35(1).

23. Rashid S, Labani S, Das BC. Knowledge, awareness and attitude on HPV, HPV vaccine and cervical cancer among the college students in India. PloS one 2016; 11(11).

24. Hussain S, Nasare V, Kumari M. Perception of human papillomavirus infection, cervical cancer and HPV vaccination in North Indian population. PLoS One 2014; 9(11): e112861.
25. Chaudhuri S, Muduli A, Arora R. Family Roles Posing Challenges for Women Leaders in India. Indian Women in Leadership. Springer 2018: 13-29.

26. Chowdhury A, Patnaik MM. Understanding Indian family life: The gender perspectives. EXCEL International Journal of Multidisciplinary Management Studies 2013; 3(7): 58-67.

27. Tripathy S, Mohapatra S, Muthulakshmi M et al. Knowledge, attitude towards human papillomavirus and HPV vaccine among medical students of a tertiary care teaching hospital in India. Int J Reprod Contracept Obstet Gynecol 2015; 4(6): 1771-1774.

28. Krupp K, Marlow LA, Kielmann KI. Factors associated with intention-to-recommend human papillomavirus vaccination among physicians in Mysore, India. Journal of adolescent health 2010; 46(4): 379-384.

29. Majumdar C. Attitudes towards premarital sex in India: Traditionalism and cultural change. Sexuality \& Culture 2018; 22(2): 614-631.

30. Jain N, Singhal M. Sexual Communication and Attitudes toward Sexual and Reproductive Health of ParentAdolescent Dyads. Journal of Indian Association for Child \& Adolescent Mental Health 2017; 13(4).

31. Wong LP. Role of men in promoting the uptake of HPV vaccinations: focus groups' finding from a developing country. International Journal of Public Health 2010; 55(1): 35-42.

32. Madhivanan P, Pierre-Victor D, Mukherjee S et al. Human papillomavirus vaccination and sexual disinhibition in females: a systematic review. American journal of preventive medicine 2016; 51(3): 373-383. 